\title{
CONSORT 2010: Aktualisierte Leitlinie für Berichte randomisierter Studien im Parallelgruppen-Design
}

\author{
K. F. Schulz 1 \\ D. G. Altman 2 \\ D. Moher ${ }^{3}$
}

Medizinisches Publizieren

Institut

${ }^{1}$ Family Health International, Research Triangle Park, North Carolina, United States of

America

${ }^{2}$ Centre for Statistics in Medicine University of Oxford, Wolfson

College, Oxford, United Kingdom ${ }^{3}$ Ottawa Methods Centre, Clinical Epidemiology Program, Ottawa Hospital Research Institute, Department of Epidemiology and Community Medicine, University of Ottawa, Ottawa, Canada

Bibliografie

Dol $10.1055 / \mathrm{s}-0031-1272980$

Dtsch Med Wochenschr 2011;

136: e20-e23 - ( ) Georg Thieme Verlag KG Stuttgart · New York · ISSN 0012-0472

Korrespondenz

Dr. Gerd Antes

Deutsches Cochrane Zentrum Abteilung für Medizinische Biometrie und Statistik Institut für Medizinische Biometrie und Medizinische Informatik

Universitätsklinikum Freiburg

Berliner Allee 29

79110 Freiburg

eMail antes@cochrane.de
Bearbeitete Übersetzung aus dem

Englischen:

Max H Pittler MD PhD, 1

Anette Blümle PhD, 1

Joerg J MeerpohI MD, 1, 2

Gerd Antes PhD ${ }^{1}$

Deutsches Cochrane Zentrum, Abteilung

für Medizinische Biometrie und Statistik,

Universitätsklinikum Freiburg, Freiburg

2Pädiatrische Hämatologie und Onkologie,

Zentrum für Kinder- und Jugendmedizin, Universitätsklinikum Freiburg, Freiburg

\section{Einleitung}

1996 wurde mit dem CONSORT Statement („Consolidated Statement of Reporting Trials“) erstmals eine Leitlinie für Veröffentlichungen von randomisierten Therapiestudien verfasst, deren aktualisierte Version von 2001 bereits ins Deutsche übersetzt wurde [1,15]. 2010 wurde eine weitere Aktualisierung notwendig, die parallel in mehreren englischsprachigen Fachzeitschriften veröffentlicht wurde [20]. Das CONSORT 2010 Statement gibt Autoren Empfehlungen für die Erstellung von Berichten von randomisierten kontrollierten Studien in Form einer Checkliste (Tab. 1) und eines Flussdiagramms ( Abb. 1) und soll damit zur Verbesserung der Berichterstattung von klinischen Studien beitragen. In einem ausführlicheren Artikel „CONSORT 2010 explanation and elaboration“ sind umfassende Erklärungen und Erläuterungen zu CON-
SORT 2010 enthalten [14]. Derzeit wird das CONSORT 2010 Statement von über 400 Zeitschriften explizit unterstützt und indirekt von Tausenden durch die Unterstützung der „Uniform Requirement for Manuscripts submitted to Biomedical Journals“ des International Committee of Medical Journal Editors (www.icmje.org). Im Folgenden ist diese aktualisierte Fassung des CONSORT Statements von Mitarbeitern des Deutschen Cochrane Zentrums am Universitätsklinikum Freiburg in Absprache mit den Autoren der Originalpublikation in ihren wesentlichen Punkten ins Deutsche übersetzt worden.

\section{Hintergrund und Ziel}

Randomisierte kontrollierte Studien sind, wenn sie angemessen geplant, durchgeführt und berichtet sind, der Goldstandard zur Bewertung medizinischer Interventionen. Jedoch können auch randomisierte Studien zu verzerrten Ergebnissen führen, wenn diese methodisch nicht sorgfältig durchgeführt werden. Um die Ergebnisse von Studienberichten genau beurteilen zu können, benötigt der Leser vollständige, klare und transparente Informationen zu Methodik und Ergebnissen. Häufig versäumen es Studienautoren jedoch, die wesentlichen Informationen verständlich und vollständig darzustellen. Inadäquate Studienbeschreibungen führten 1996 zur Entwicklung des CONSORT Statements und zu seiner 5 Jahre später publizierten Weiterentwicklung. Das aktualisierte CONSORT 2010 Statement enthält eine 25-Punkte-Checkliste so-

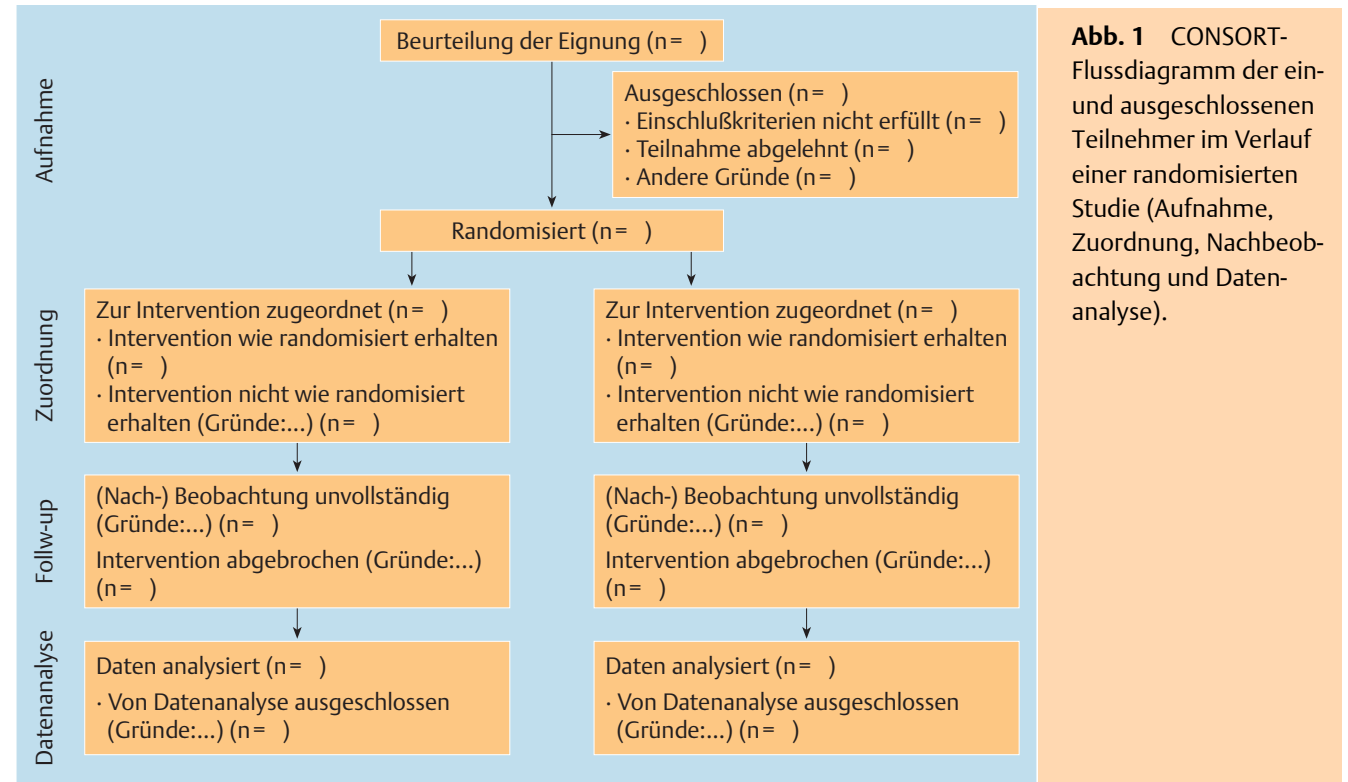


Tab. 1 CONSORT-Checkliste einzuschließender Informationen in Berichten über randomisierte Studien*.

\begin{tabular}{|c|c|c|}
\hline Abschnitt/Thema & Nummer & Beschreibung \\
\hline \multicolumn{3}{|l|}{ Titel und Zusammenfassung } \\
\hline & $1 \mathrm{a}$ & Kennzeichnung im Titel als randomisierte Studie \\
\hline & $1 \mathrm{~b}$ & $\begin{array}{l}\text { Strukturierte Zusammenfassung von Studiendesign, Methoden, Resultaten und Schlussfolgerungen } \\
\text { (siehe auch CONSORT für Abstracts }[10,11] \text { ) }\end{array}$ \\
\hline \multicolumn{3}{|l|}{ Einleitung } \\
\hline \multirow[t]{2}{*}{ Hintergrund und Ziele } & $2 a$ & Wissenschaftlicher Hintergrund und Begründung der Studie \\
\hline & $2 b$ & Genaue Fragestellung oder Hypothesen \\
\hline \multicolumn{3}{|l|}{ Methoden } \\
\hline \multirow[t]{2}{*}{ Studiendesign } & $3 a$ & $\begin{array}{l}\text { Beschreibung des Studiendesigns (z. B. parallel, faktoriell), einschließlich Zuteilungsverhältnis der Patienten } \\
\text { zu den Gruppen }\end{array}$ \\
\hline & $3 b$ & Wichtige Änderungen der Methoden nach Studienbeginn (z.B. Eignungskriterien) mit Gründen \\
\hline \multirow[t]{2}{*}{ Probanden/Patienten } & $4 a$ & Eignungskriterien der Probanden/Patienten \\
\hline & $4 \mathrm{~b}$ & Umgebung und Ort der Studiendurchführung \\
\hline Intervention/Behandlung & 5 & $\begin{array}{l}\text { Durchgeführte Interventionen in jeder Gruppe mit präzisen Details, einschließlich wie und wann die } \\
\text { Interventionen durchgeführt wurden, um eine Replikation der Studie zu ermöglichen }\end{array}$ \\
\hline \multirow[t]{2}{*}{ Endpunkte } & $6 a$ & $\begin{array}{l}\text { Vollständig definierte, primäre und sekundäre Endpunkte (früher „Zielkriterien“ genannt), } \\
\text { einschließlich wie und wann sie erhoben wurden }\end{array}$ \\
\hline & $6 b$ & Änderungen der Endpunkte nach Studienbeginn mit Angabe der Gründe \\
\hline \multirow[t]{2}{*}{ Fallzahlbestimmung } & $7 a$ & Wie wurde die Fallzahl berechnet? \\
\hline & 7b & Falls zutreffend, Erklärung aller Zwischenanalysen und Abbruchkriterien \\
\hline \multicolumn{3}{|l|}{ Randomisierung } \\
\hline \multirow[t]{2}{*}{ Erzeugung der Behandlungsfolge } & $8 a$ & Methode zur Generierung der Zufallszuteilung \\
\hline & $8 \mathrm{~b}$ & Art der Randomisierung; Details jedweder Restriktionen (z.B. Blockbildung, Blockgröße) \\
\hline $\begin{array}{l}\text { Mechanismen der Geheimhal- } \\
\text { tung der Behandlungsfolge }\end{array}$ & 9 & $\begin{array}{l}\text { Mechanismen zur Umsetzung der Zuteilungssequenz (z.B. sequenziell nummerierte Behälter) und } \\
\text { Beschreibung aller Schritte zur Geheimhaltung der Sequenz bis zur Interventionszuordnung }\end{array}$ \\
\hline Durchführung & 10 & $\begin{array}{l}\text { Wer führte die Zufallszuteilung durch, wer nahm die Teilnehmer in die Studie auf und wer teilte die } \\
\text { Teilnehmer den Interventionen zu }\end{array}$ \\
\hline \multirow[t]{2}{*}{ Verblindung } & $11 \mathrm{a}$ & $\begin{array}{l}\text { Falls durchgeführt, wer war bei der Interventionszuordnung verblindet? (Z.B. Teilnehmer, Ärzte, } \\
\text { Therapeuten, diejenigen, die die Endpunkte beurteilten) }\end{array}$ \\
\hline & $11 b$ & Falls relevant, Beschreibung der Ähnlichkeit der Interventionen \\
\hline \multirow[t]{2}{*}{ Statistische Methoden } & $12 \mathrm{a}$ & $\begin{array}{l}\text { Statistische Methoden, die zum Vergleich der Gruppen hinsichtlich primärer und sekundärer Endpunkte } \\
\text { eingesetzt wurden }\end{array}$ \\
\hline & $12 \mathrm{~b}$ & Methoden, die für zusätzliche Analysen eingesetzt wurden, wie Subgruppenanalysen, adjustierte Analysen \\
\hline \multicolumn{3}{|l|}{ Ergebnisse } \\
\hline \multirow[t]{2}{*}{$\begin{array}{l}\text { Ein- und Ausschlüsse (ein Flussdia- } \\
\text { gramm wird dringend empfohlen) }\end{array}$} & $13 a$ & $\begin{array}{l}\text { Für jede Gruppe Anzahl der Studienteilnehmer, die randomisiert zugeteilt wurden, die die geplante } \\
\text { Intervention erhielten und die hinsichtlich des primären Endpunkts analysiert wurden }\end{array}$ \\
\hline & $13 \mathrm{~b}$ & Für jede Gruppe Zahl der Studienausscheider und Ausschlüsse nach Randomisierung mit Angabe von Gründen \\
\hline \multirow[t]{2}{*}{ Aufnahme/Rekrutierung } & $14 a$ & Zeitraum der Rekrutierung und Nachbeobachtung \\
\hline & $14 b$ & Warum die Studie endete oder gestoppt wurde \\
\hline $\begin{array}{l}\text { Patientencharakteristika zu } \\
\text { Studienbeginn (baseline data) }\end{array}$ & 15 & Eine Tabelle demographischer und klinischer Charakteristika für jede Gruppe \\
\hline $\begin{array}{l}\text { Anzahl der ausgewerteten } \\
\text { Probanden/Patienten }\end{array}$ & 16 & $\begin{array}{l}\text { Für jede Gruppe, Anzahl der Teilnehmer, die in die Analyse eingeschlossen wurde und Angabe, } \\
\text { ob diese der Anzahl der ursprünglich zugeteilten Gruppen entsprach }\end{array}$ \\
\hline \multirow[t]{2}{*}{$\begin{array}{l}\text { Ergebnisse und } \\
\text { Schätzmethoden }\end{array}$} & $17 a$ & $\begin{array}{l}\text { Für jeden primären und sekundären Endpunkt Ergebnisse für jede Gruppe und die geschätzte Effektgröße sowie } \\
\text { ihre Präzision (z.B. 95\%-Konfidenzintervall) }\end{array}$ \\
\hline & $17 \mathrm{~b}$ & Für binäre Endpunkte wird empfohlen, sowohl die absoluten als auch die relativen Effektgrößen anzugeben \\
\hline Zusätzliche Analysen & 18 & $\begin{array}{l}\text { Resultate von weiteren Analysen, einschließlich Subgruppenanalysen und adjustierten Analysen mit Angabe, } \\
\text { ob diese präspezifiziert oder exploratorisch durchgeführt wurden }\end{array}$ \\
\hline Schaden & 19 & Alle wichtigen Schäden (früher „unerwünschte Wirkungen“ genannt) innerhalb jeder Gruppe (siehe CONSORT für Schäden [12]) \\
\hline \multicolumn{3}{|l|}{ Diskussion } \\
\hline Limitierungen & 20 & Studienlimitierungen mit Angabe zu potentieller Verzerrung, fehlender Präzision und, falls relevant, Multiplizität von Analysen \\
\hline Generalisierbarkeit & 21 & Generalisierbarkeit (externe Validität, Anwendbarkeit) der Studienergebnisse \\
\hline Interpretation & 22 & $\begin{array}{l}\text { Interpretation konsistent mit den Ergebnissen, Abwägung des Nutzens und Schadens, } \\
\text { Berücksichtigung anderer relevanter Evidenz }\end{array}$ \\
\hline \multicolumn{3}{|l|}{ Andere Information } \\
\hline Registrierung & 23 & Registrierungsnummer und Name des Studienregisters \\
\hline Protokoll & 24 & Wo das vollständige Protokoll eingesehen werden kann, falls verfügbar \\
\hline Finanzierung & 25 & Quellen der Finanzierung und anderer Unterstützung (wie Lieferung von Medikamenten), Rolle des Geldgebers \\
\hline
\end{tabular}


Punkt 1b (Titel und Zusammenfassung): Hinzugefügt wurde ein Unterpunkt zur Bereitstellung einer strukturierten Zusammenfassung von Studiendesign, Methoden, Ergebnissen und Schlussfolgerung sowie die Referenz von CONSORT für Abstracts [10].

Punkt 2b (Einleitung): Ein neuer Unterpunkt (ehemals Punkt 5 in CONSORT 2001) zu „Genaue Fragestellungen und Hypothesen“ wurde hinzugefügt.

Punkt 3a (Studiendesign): Ein neuer Punkt, dazu zählt auch dieser Unterpunkt, wurde hinzugefügt, um das Studiendesign (z.B. parallel, cross-over, Cluster) und das Zuteilungsverhältnis zu den Gruppen klar darzulegen.

Punkt 3b (Studiendesign): Hinzugefügt wurde ein neuer Unterpunkt zu allen wichtigen Änderungen der Studienmethoden, die nach Beginn der Studie durchgeführt wurden, inkl. Darstellung der Gründe.

Punkt 4 (Probanden/Patienten): Ehemals Punkt 3 in CONSORT 2001.

Punkt 5 (Intervention/Behandlung): Ehemals Punkt 4 in CONSORT 2001. Es wird größere Spezifität gefordert durch die Angabe, dass Beschreibungen von Interventionen genügend Angaben beinhalten sollten, um eine Wiederholung zu ermöglichen [8]. Punkt 6 (Endpunkte): Hinzugefügt wurde ein Unterpunkt, um alle Änderungen hinsichtlich der primären und sekundären Endpunkte (früher „Zielkriterien“ genannt), die nach Beginn der Studie durchgeführt wurden, kenntlich zu machen. Empirische Untersuchungen zeigen, dass Autoren häufig Analysen von Endpunkten beschreiben, die in den Studienprotokollen nicht als primäre und sekundäre Endpunkte vordefiniert sind, während vordefinierte Endpunkte ignoriert werden (d.h. selektive Berichterstattung von Endpunkten) [4,6]. Gestrichen wurde Text bzgl. jeglicher Methoden, die sich auf die Verbesserung der Qualität der Messungen bezogen.

Punkt 9 (Mechanismen zur Geheimhaltung der Behandlungsfolge): Dieser Punkt wurde umformuliert, um „Mechanismen“ mit aufzunehmen; hierdurch wird bekräftigt, dass Autoren die tatsächlich durchgeführten Schritte zur Gewährleistung der Geheimhaltung der Behandlungsfolge berichten sollen, anstatt einfach nur unpräzise Zusicherungen zu der erfolgten Verdeckung zu geben.

Punkt 11 (Verblindung): Es wurde eine Spezifikation, d. h. wie die Verblindung durchgeführt wurde, hinzugefügt und, falls relevant, eine Beschreibung über Ähnlichkeit der Interventionen und Maßnahmen. Aufgrund fehlender unterstützender empirischer Untersuchungen sowie theoretischer Bedenken gegenüber der Validität solcher Erhebungen wurde außerdem der Text „Wie wurde der Erfolg der Verblindung evaluiert" gestrichen [19, 21].

Punkt 12a (Statistische Methoden): Hinzugefügt wurde, dass auch statistische Methoden für Analysen von sekundären Endpunkten beschrieben werden sollten

Unterpunkt 14b (Aufnahme/Rekrutierung): Basierend auf empirischen Untersuchungen wurde der Unterpunkt „Warum die Studie endete oder gestoppt wurde“ hinzugefügt [16].

Punkt 15 (Patientencharakteristika zu Studienbeginn): Dieser Punkt wurde durch das Hinzufügen von „eine Tabelle“ genauer spezifiziert, um zu verdeutlichen, dass sowohl Patienten- als auch klinische Charakteristika jeder Gruppe in einer Tabelle am deutlichsten dargestellt werden können.

Punkt 16 (Anzahl der ausgewerteten Probanden/Patienten): , Intention to treat' - ein oft falsch verwendeter Begriff - wurde gegen eine ausdrücklichere Forderung nach Information über die Beibehaltung der Teilnehmer in ihren ursprünglich zugeordneten Gruppen ersetzt [9].

Unterpunkt 17b (Ergebnisse und Schätzmethoden): Für eine angemessene klinische Interpretierbarkeit legte es die allgemeine Erfahrung nahe, für binäre Endpunkte die Darstellung sowohl von relativen als auch absoluten Effektgrößen zu empfehlen [17]. Punkt 19 (Schaden): Hinzugefügt wurde die Referenz des CONSORT-Artikels zu Schaden (früher „unerwünschte Wirkungen“ genannt) [12].

Punkt 20 (Limitierungen): Der frühere Punkt „Interpretation“ wurde ersetzt und durch einen Satz ergänzt, der auf die Berichterstattung von Ursachen potentieller Verzerrungen und Ungenauigkeiten fokussiert.

Punkt 22 (Interpretation): Der frühere Punkt „Bewertung der Evidenz“ wurde geändert. Zwar sollte mit dieser nebulösen Überschrift den Autoren Spielraum für die Interpretation zugestanden werden, jedoch äußerte die CONSORT-Gruppe Bedenken, dass Schlussfolgerungen von Artikeln oft den tatsächlichen analytischen Ergebnisse nicht entsprechen und Schäden ignoriert oder als unbedeutsam bewertet werden. Deshalb wurde dieser Punkt der Checkliste geändert, um das Konzept aufzunehmen, dass Ergebnisse mit den Interpretationen übereinstimmen und Nutzen und Schaden abgewogen werden.

Punkt 23 (Registrierung): Neu hinzugefügt wurde dieser Punkt zur Studienregistrierung. Empirische Untersuchungen unterstützen die Notwendigkeit der Studienregistrierung, und aktuelle Anforderungen von Zeitschrifteneditoren fördern die Zustimmung [5]. Punkt 24 (Protokoll): Neu hinzugefügt wurde dieser Punkt zur Verfügbarkeit eines Studienprotokolls. Empirische Untersuchungen weisen darauf hin, dass Autoren bei der Durchführung und Berichterstattung ihrer Studie oft nicht berücksichtigen, was im Protokoll angegeben wurde $[4,6]$. Wird das Studienprotokoll öffentlich verfügbar gemacht, kann das helfen, dass das Protokoll während der Studiendurchführung besser eingehalten wird. Ebenso erleichtert es nach der Publikation die Beurteilung über die Einhaltung des Protokolls.

Punkt 25 (Finanzierung): Neu hinzugefügt wurde dieser Punkt zur Finanzierung. Empirische Untersuchungen zeigen auf, dass die Finanzierungsquellen manchmal mit den geschätzten Behandlungseffekten assoziiert sind [13].

wie ein Flussdiagramm und stellt eine Leitlinie für Berichte über randomisierte Studien jeglicher Art dar, fokussiert aber auf das häufigste Studiendesign, individuell randomisierte Parallelvergleiche zweier Gruppen. Andere Studientypen wie z.B. „Cluster“-randomisierte Studien erfordern in unterschiedlichem Umfang zusätzliche Informationen $[3,18]$. Die CONSORT-Erweiterungen für diese Studi- entypen, sowie weitere Informationen zu CONSORT, sind unter www.consortstatement.org abrufbar. Zusammen mit dem CONSORT Statement wurde auch der ausführliche Artikel „CONSORT ex- 
planation and elaboration" aktualisiert, in welchem jeder einzelne Punkt der Checkliste erklärt wird und der den methodischen Hintergrund sowie Beispiele für transparente Berichterstattung liefert [14]. Gewissenhafte Einhaltung der Checklistenpunkte erleichtert vollständiges, klares und transparentes Berichten. CONSORT 2010 enthält keine Empfehlungen zu Design, Durchführung oder Analyse von Studien. Einzig das Berichten von Studien, also was gemacht wurde und was gefunden wurde, wird angesprochen.

Dennoch ist anzunehmen, dass CONSORT indirekt auch das Design und die Durchführung einer Studie beeinflusst. Transparentes Berichten deckt Mängel in der Forschung auf, falls solche vorhanden sind. Folglich sollte es nicht möglich sein, dass Wissenschaftler, die methodisch unzureichende Studien durchführen, dies jedoch transparent berichten müssen, den Publikationsprozess durchlaufen können, ohne dass diese Unzulänglichkeiten aufgedeckt werden.

\section{Ånderungen}

$\checkmark$

Das aktualisierte CONSORT 2010 Statement ist eine Weiterentwicklung basierend auf Erfahrungen im Umgang mit CONSORT 2001. Allgemeine Änderungen betreffen zum einen einige Punkte der Checkliste, welche vereinfacht und präziser formuliert wurden, zum anderen wurde auf einen konsistenten Stil der Formulierungen geachtet, indem auf Verben im Imperativ, die in der Version von 2001 verwendet wurden, verzichtet wurde. Desweiteren wurden einige Punkte der Checkliste in Unterpunkte aufgegliedert. Diese spezifischen Änderungen sind in Box 1 dargestellt.

\section{Limitierungen}

$\nabla$

Das CONSORT Statement ist entwickelt worden, um Autoren, Redakteuren und Gutachtern beim Schreiben, Editieren und Beurteilen von Manuskripten sowie Lesern bei der kritischen Beurteilung eines Studienberichts zu helfen. Der ausführliche Artikel „CONSORT 2010 explanation and elaboration“ bietet zusätzliche Erläuterungen zu den einzelnen Punkten der Checkliste. Es wird daher dringend geraten, diesen in Verbindung mit der Checkliste zu nutzen, um eine klare und transparente Berichterstat- tung zu fördern und die Bewertung von publizierten Studienberichten zu unterstützen. Zwar wurde CONSORT 2010 hauptsächlich für randomisierte Studien mit zwei parallelen Gruppen entwickelt, doch können die meisten Punkte der Checkliste auch auf andere Typen randomisierter Studien angewendet werden. Nichtsdestotrotz erfordern einige Studientypen oder Studiensituationen zusätzliche Angaben in den Studienberichten. Im Zweifelsfall sollte die CONSORT-Internetseite (siehe oben) oder auch das Equator-Netzwerk („Enhancing the Quality and Transparency of Health Research“: www.equator-network.org) hinzugezogen werden.

CONSORT 2010 gibt absichtlich keine rigide Struktur für Berichte über randomisierte Studien vor. Vielmehr sollten Artikel dem Stil des Journals, redaktionellen Direktiven und Traditionen des Forschungsbereiches und, wo möglich, Autorenpräferenzen folgen. Es ist nicht erwünscht, die Struktur des Berichtens zu standardisierten. CONSORT zielt auf das vollständige, klare und transparente Berichten, das das tatsächliche Studiendesign und die Durchführung widerspiegelt. CONSORT 2010 ist nicht als ein Instrument zur Qualitätsbewertung von Studien gedacht. Auch sollte die Checkliste nicht als Grundlage von Qualitätsskalen verwendet werden. Die breite Unterstützung von CONSORT durch Zeitschriften und Redakteure sollte dazu führen, dass die meisten Autoren alle wichtigen Studienaspekte transparent berichten (Tab. 1).

Autorenerklärung: Die Autoren erklären, dass sie keine finanziellen Verbindungen mit einer Firma haben, deren Produkt in dem Artikel eine wichtige Rolle spielt (oder mit einer Firma, die ein Konkurrenzprodukt vertreibt).

\section{Literatur}

1 Begg C, Cho M, Eastwood S et al. Improving the quality of reporting of randomized controlled trials. The CONSORT statement. JAMA 1996; 276: 637-639

2 Boutron I, Moher D, Altman DG, Schulz KF, Ravaud $P$. Extending the CONSORT statement to randomized trials of nonpharmacologic treatment: explanation and elaboration. Ann Intern Med 2008; 148: 295-309

3 Campbell MK, Elbourne DR, Altman DG. CONSORT statement: extension to cluster randomised trials. BMJ 2004; 328: 702-708

4 Chan AW, Hrobjartsson A, Haahr MT, Gotzsche $P C$, Altman DG. Empirical evidence for selective reporting of outcomes in randomized trials: comparison of protocols to published articles. JAMA 2004; 291: 2457-2465
5 De Angelis C, Drazen JM, Frizelle FA et al. Clinical trial registration: a statement from the International Committee of Medical Journal Editors. Ann Intern Med 2004; 141: 477-478

6 Dwan K, Altman DG, Arnaiz JA et al. Systematic review of the empirical evidence of study publication bias and outcome reporting bias. PLoS ONE 2008; 3: e3081-

7 Gagnier JJ, Boon H, Rochon P et al. Reporting randomized, controlled trials of herbal interventions: an elaborated CONSORT statement. Ann Intern Med 2006; 144: 364-367

8 Glasziou P, Meats E, Heneghan C, Shepperd S. What is missing from descriptions of treatment in trials and reviews? BMJ 2008; 336: 1472-1474

9 Hollis $S$, Campbell $F$. What is meant by intention to treat analysis? Survey of published randomised controlled trials. BMJ 1999; 319: 670-674

10 Hopewell S, Clarke M, Moher D et al. CONSORT for reporting randomised trials in journal and conference abstracts. Lancet 2008; 371: 281-283

11 Hopewell S, Clarke M, Moher D et al. CONSORT for reporting randomized controlled trials in journal and conference abstracts: explanation and elaboration. PLoS Med 2008; 5: e20-

12 Ioannidis JP, Evans SJ, Gotzsche PC et al. Better reporting of harms in randomized trials: an extension of the CONSORT statement. Ann Intern Med 2004; 141: 781-788

13 Lexchin J, Bero LA, Djulbegovic B, Clark $O$. Pharmaceutical industry sponsorship and research outcome and quality: systematic review. BMJ 2003; 326: 1167-1170

14 Moher D, Hopewell S, Schulz KF et al. CONSORT 2010 explanation and elaboration: updated guidelines for reporting parallel group randomised trials. BMJ 2010; 340: c869-

15 Moher D, Schulz KF, Altman DG, Gruppe fdC. Das CONSORT Statement: Überarbeitete Empfehlungen zur Qualitätsverbesserung von Reports randomisierter Studien im Parallel-Design. Dtsch med Wochenschr 2004; 129: T16-T20

16 Montori VM, Devereaux PJ, Adhikari NK et al. Randomized trials stopped early for benefit: a systematic review. JAMA 2005; 294: 2203 2209

17 Nuovo J, Melnikow J, Chang D. Reporting number needed to treat and absolute risk reduction in randomized controlled trials. JAMA 2002; 287: 2813-2814

18 Piaggio G, Elbourne DR, Altman DG, Pocock SJ, Evans SJ. Reporting of noninferiority and equivalence randomized trials: an extension of the CONSORT statement. JAMA 2006; 295: $1152-1160$

19 Sackett DL. Commentary: Measuring the success of blinding in RCTs: don't, must, can't or needn't? Int J Epidemiol 2007; 36: 664-665

20 Schulz KF, Altman DG, Moher D for the CONSORT Group. CONSORT 2010 Statement: updated guidelines for reporting parallel group randomised trials. BMJ 2010; 340: c332-

21 Schulz KF, Grimes DA. Blinding in randomised trials: hiding who got what. The Lancet 2002; 359: 696-700

22 Zwarenstein $M$, Treweek S, Gagnier JJ et al. Improving the reporting of pragmatic trials: an extension of the CONSORT statement. BMJ 2008; 337: a2390 